\title{
LINK BETWEEN AUTOIMMUNE HYPOTHYROIDISM AND POLYCYSTIC OVARY SYNDROME.
}

\author{
1. MBBS, M.Phil \\ Assistant Professor Physiology \\ Aziz Fatimah Medical and \\ Dental College, Faisalabad. \\ 2. MBBS, M.Phil \\ Associate Professor Physiology \\ Aziz Fatimah Medical and \\ Dental College, Faisalabad. \\ 3. Ph.D \\ Assistant Professor IMBB \\ University of Lahore, Lahore.
}

Correspondence Address:

Dr. Anam Rehman

Department of Physiology

Aziz Fatimah Medical and

Dental College, Faisalabad.

doc.rehman158@gmail.com

Article received on:

28/05/2019

Accepted for publication:

29/04/2020

\begin{abstract}
Anam Rehman 1 , Shireen Jawed ${ }^{2}$, Amna Rashid Tariq ${ }^{3}$
ABSTRACT... Objectives: Polycystic ovary syndrome (PCOS) is a rampant endocrine disorder distressing women of child bearing age worldwide. Many current researches have detected the presence of some organ specific and non-specific autoantibodies in females with PCOS. Study Design: Cross Sectional study. Setting: Aziz Fatimah Hospital, Faisalabad, Pakistan. Period: April to September 2017. Material \& Methods: This study comprised of 88 female subjects of 17-35 years old. Participants were divided into four group's i.e PCOS obese females, PCOS non-obese, obese females without PCOS and age matched controls. Thyroid function was evaluated by the measurement of serum TSH, FT3 and FT4 levels. Thyroid peroxidase antibody (TPO-Antibody) was detected as an indicator of thyroid autoimmunity. All parameters were measured by chemiluminescence immunoassay technique (CLIA). SPSS version 22 was used for the statistical analysis of the data. Results: Out of total 88 female participants, $38.6 \%$ were hypothyroid and $61.4 \%$ were euthyroid females. While on comparing the percentages of hypothyroidism among the study groups PCOS, non-PCOS patients and obese we found higher percentages of hypothyroidism among non-obese PCOS. Thyroid peroxidase antibody levels were higher in PCOS obese subjects. PCOS patients have 15 times more risk for hypothyroidism as compared to non-PCOS patients. Conclusion: Hypothyroidism was commonly found in PCOS patients with high levels of TPO-Antibody indicating that PCOS is an independent risk factor for hypothyroidism which suggests that evaluation of thyroid function and autoimmunity must be deliberated in PCOS patients.
\end{abstract}

Key words: Hypothyroidism, Obesity, PCOS, Thyroid Autoimmunity.

Article Citation: Rehman A, Jawed S, Tariq AR. Link between Autoimmune Hypothyroidism and Polycystic Ovary Syndrome. Professional Med J 2020; 27(9):18041808. DOI: 10.29309/TPMJ/2020.27.09.3750

\section{INTRODUCTION}

The Polycystic ovary syndrome (PCOS) is a metabolic syndrome which is characterized by anovulation, hyperandrogenism and polycystic ovary. ${ }^{1}$ The prevalence of PCOS is $3 \%$ to $15 \%$ according to the studied communities. ${ }^{2}$ PCOS is also associated with the increasing rates of loss of pregnancy. It is one of the most common reasons of infertility in women of child bearing age $^{3}$.

Regardless of the extensive studies on PCOS, the exact underlying pathogenesis is yet to be discovered. It is considered to be a heterogeneous disorder with both hereditary and the ecological components. ${ }^{3,4}$

Strong evidences are available showing that the PCOS patients possess high risk of endocrine and metabolic diseases including thyroid abnormalities, diabetes mellitus due to decreased tolerance to glucose and obesity. Studies regarding the underlying pathogenesis of PCOS are mainly concerned with two related metabolic elements that is the resistance to insulin and hyperandrogenism. ${ }^{5}$

The autoimmune thyroid diseases (AITD) are the typical autoimmune disorders which affect almost $5 \%$ to $20 \%$ of females in the reproductive age. Thyroid autoimmunity is the commonest reason of hypothyroidism among young females. It might be existent without thyroid dysfunction for a long time, hence, getting ignored and resulting in hypothyroidism further down the road. ${ }^{4,6}$ The most sensitive marker of thyroid autoimmunity is the high levels of thyroid peroxidase autoantibodies 
(TPO-Abs) which are related with hypothyroidism as well as hyperthyroidism. ${ }^{6}$

The autoimmune thyroiditis or the Hashimoto's thyroiditis is the utmost common form of thyroid gland which is characterized by diffused lymphocytic infiltration, usually, followed by hypothyroidism. It is believed that it is because of the destruction and ultimate fibrous restoration of the parenchymal tissue. ${ }^{7}$ Several females with thyroid dysfunction encounter extreme irregular menstruation, lessened fertility rate and raised risk for spontaneous abortion as well as the preterm births. ${ }^{8}$ Link between PCOS, thyroid autoimmunity and obesity are documented in the previous studies but exact relation is still debatable upon due to controversial results. ${ }^{9}$

Elevated prolactin level secondary to increase Thyrotropin releasing hormone (TRH) due to hypothyroidism cause polycystic appearance of ovary by hindering the formation of an egg which alters the ratio of follicle stimulating hormone $(\mathrm{FSH})$ and luteinizing hormone $(\mathrm{LH})$ as well as elevated level of dehydroepiandrosterone. ${ }^{10,11,12}$ High concentrations of thyroid stimulating hormone (TSH) in hypothyroidism might be responsible for hyperstimulation of ovaries by activation of follicle stimulating hormone receptor (FSHR). ${ }^{11}$

The concurrence of hypothyroidism and PCOS are associated with complicated pathophysiological changes caused by obesity and insulin resistance in PCOS. ${ }^{11}$ Previous studies are reported that $44 \%$ of females with polycystic ovary syndrome are obese and have deteriorating effects. This figure differs to a certain extent depending on the ethnicity and geography. It aggravate the clinical presentation of PCOS by increasing resistance to insulin and leading to elevated levels of ovarian estrogen along with the raised adrenal androgens and the unbound testosterone. ${ }^{11}$ The timely diagnosis and management of hypothyroidism and obesity in PCOS might decrease the frequency of infertility and complications related to pregnancy in these females.

\section{Aim}

This study was aimed to find the frequency of hypothyroidism among obese PCOS and nonobese PCOS and to compare it among nonPCOS obese and controls. We also aimed to explore the association between PCOS and thyroid peroxidase antibodies.

\section{MATERIAL \& METHODS}

This cross sectional study was conducted during April to September 2017 at Aziz Fatimah Hospital, Faisalabad, Pakistan. It comprised of 88 female subjects of 17-35 years old. Participants were divided into four groups i.e PCOS obese females, PCOS non-obese, obese females without PCOS and age matched control subjects. Diagnosed PCOS subjects were enrolled from the outpatient department of gynecology/obstetrics, obese and controls were recruited from nutrition department by non-probability purposive sampling technique.

Females were categorized as PCOS as per Rotterdam criteria 2003. According to this criteria patients having two out of the three features including: (a) oligomenorrhea or amenorrhea, (b) hyperandrogenism (raised androgen levels), (c) polycystic ovaries on ultrasound were labeled as PCOS. ${ }^{13}$ Age matched control females having BMl less than $25 \mathrm{~kg} / \mathrm{m}^{2}$ with regular menstrual cycle, no sign of hyperandrogenism along with normal ovaries appearing on ultrasound were taken. Subjects having BMI greater than $25 \mathrm{~kg} / \mathrm{m}^{2}$ with regular menstrual cycle and normal ovaries were taken as obese. Females with diagnosed hypothyroidism and on treatment with thyroxine were excluded. Subjects with tumor of pituitary gland or ovary and with the previous history of neck and pelvic surgery were excluded. The unwilling females were also excluded.

Informed consent was taken from the each participant and confidentiality was assured prior to study. A detailed history regarding fertility and menstruation and the anthropometric measurements were also taken. Height in meters and weight in $\mathrm{Kg}$ were measured by stadiometer and BMI was calculated by formula weight in $\mathrm{Kg} /$ height in meter. ${ }^{2}$ Each participant was screened for hypothyroidism. For this purpose, 4cc blood sample was drawn under the aseptic conditions 
of each participant. Serum was separated from blood by centrifuging and stored at $-20^{\circ} \mathrm{C}$ in the pathology laboratory of Aziz Fatimah Hospital for further analysis. Thyroid stimulating hormone (TSH), free tri-idothyrionine (FT3), free thyroxine (FT4) and thyroid peroxidase antibodies (TPO$\mathrm{Ab})$ were measured by chemiluminescence immunoassay technique (CLIA).

\section{Statistical Analysis}

Statistical analysis was done using SPSS version 22. Data is expressed as mean and standard deviation for continuous variables (age, height, weight and BMI). Categorical variables are presented as frequency (n) and percentage (\%). X2 test was used to compare percentages (Thyroid Peroxidase Antibodies and Hypothyroidism). Independent t-test was applied to compare the means of biochemical parameters (TSH, FT3 and FT4) among hypothyroid and euthyroid. Relative risk of hypothyroidism in PCOS patients was estimated by odd ratios obtained by logistic regression analysis. $P$ value $\leq 0.05$ was taken as significant.

\section{RESULTS}

Study was comprised of 88 mean age $27.13 \pm$ 3.87 years. Descriptive of study population is described in Table-I. Mean of FT3, FT4 levels were lower in hypothyroid patients and TSH was higher in hypothyroid patients as compared to euthyroid patients. Differences were statistically significant (Table-II). Figure-1 is showing the distribution of hypothyroid patients among the study population. Figure-2 is indicating that percentages of hypothyroid patients were higher in PCOS nonobese subjects as compared to PCOS obese and obese patients. TPO antibody positive titer was higher in PCOS obese subjects (P-value $\left.0.001^{*}\right)$. Relative risk of hypothyroidism in PCOS patients was estimated by odd ratios obtained by logistic regression analysis. Odd ratios of 15.08 is showing that PCOS patients have 15 times more risk for hypothyroidism as compared to nonPCOS patients (P-value $\left.=0.000^{*}\right)$.

\begin{tabular}{|l|c|}
\hline \multicolumn{1}{|c|}{ Anthropometric Measurements } & Mean \pm SD \\
\hline Age $($ age $)$ & $27.13 \pm 3.87$ \\
\hline Height $(\mathrm{m})$ & $1.60 \pm .08$ \\
\hline Weight $(\mathrm{kg})$ & $74.86 \pm 20.31$ \\
\hline BMl $(\mathrm{kg} / \mathrm{m} 2)$ & $29.27 \pm 7.80$ \\
\hline Thyroid Stimulating Hormone $(\mathrm{mlU} / \mathrm{ml})$ & $6.90 \pm 7.29$ \\
\hline Free Tri-idothyrionine $(\mathrm{pg} / \mathrm{ml})$ & $2.22 \pm 1.02$ \\
\hline Free Thyroxine $(\mathrm{pg} / \mathrm{ml})$ & $11.05 \pm 4.09$ \\
\hline Thyroid Peroxidase Antibodies $(\mathrm{IU} / \mathrm{ml})$ & $6.01 \pm 5.17$ \\
\hline
\end{tabular}

Table-I. Descriptive statistics of studied subjects.

$$
(n=88)
$$

$\mathrm{BMI}=$ Body mass index,

$\mathrm{SD}=$ Standard. Deviation, $\mathrm{m}=$ meters,

$\mathrm{Kg}=$ (kilogram), $\mathrm{mlU} / \mathrm{L}=$ milli international units per liter, $\mathrm{pg} / \mathrm{ml}=$ picograms per milliliter, $\mathrm{IU} / \mathrm{ml}=$ international units per milliliter

\begin{tabular}{|c|c|c|c|}
\hline $\begin{array}{l}\text { Biochemical } \\
\text { Variables }\end{array}$ & $\begin{array}{l}\text { Hypothyroid } \\
\mathrm{N}=34\end{array}$ & $\begin{array}{c}\text { Non- } \\
\text { Hypothyroid } \\
\mathrm{N}=54\end{array}$ & P-Value \\
\hline & Mean $\pm S D$ & Mean $\pm S D$ & \\
\hline $\mathrm{TSH}$ & $14.06 \pm 7.20$ & $2.39 \pm 1.23$ & $0.002^{*}$ \\
\hline FT3 & $1.56 \pm .99$ & $2.64 \pm .81$ & $0.003^{*}$ \\
\hline FT4 & $8.50 \pm 4.03$ & $12.66 \pm 3.26$ & 0.000 * \\
\hline
\end{tabular}

$\mathrm{SD}=$ Standard Deviation,

$\mathrm{TSH}=$ Thyroid Stimulating Hormone,

FT3=Free Tri-idothyrionine, FT4=Free Thyroxine.

$P$ value $\leq 0.05$ was taken as significant.

\section{Distribution of Hypothyroid patients among study population}

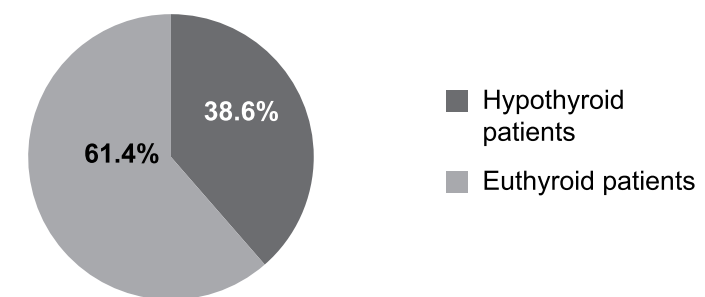

Figure-1. Distribution of hypothyroid patients among study population. 


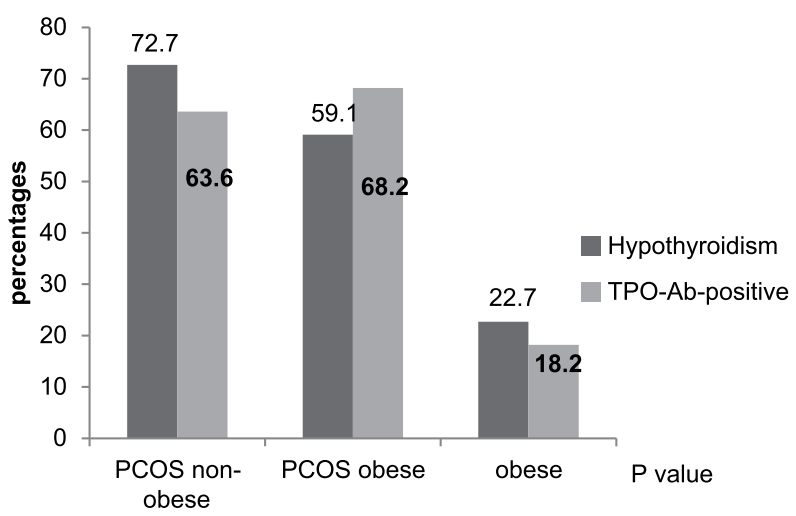

Figure-2. Hypothyroidism and positive TPO-Ab among study groups.

Chi-square test was used to compare percentages. $P$ value $\leq 0.005$ was taken as significant.

\section{DISCUSSION}

Prevalence of polycystic ovary syndrome (PCOS) and autoimmune thyroid disease is increasingly at alarming rate globally. This association is also being recognized by various researchers, but the nature of this link is still hypothesized. ${ }^{11}$

This study was conducted to highlight the emerging need to elucidate the relation between PCOS and autoimmune diseases. For this purpose we found frequency of autoimmune hypothyroidism in PCOS patients by estimating thyroid peroxidase antibodies. We also evaluate the relative risk of hypothyroidism in PCOS patients. Current study comprised of 88 subjects, out of total $38.6 \%$ were the hypothyroid patients and $61.4 \%$ are the euthyroid patients, similar figures were reported by Pushpagiri et al, who also documented significant high $\mathrm{TSH}$ and low FT3 and FT4 levels in hypothyroid patients. ${ }^{12}$ On comparing the percentages of hypothyroidism among the study groups PCOS, non-PCOS patients and obese we found higher percentages of hypothyroidism among non-obese PCOS.

The present study reveals that $72.7 \%$ are the hypothyroid patients among the non-obese PCOS while the $59.1 \%$ are the hypothyroid patients among the obese PCOS and $22.7 \%$ of obese subjects have hypothyroidism. We found significantly high thyroid peroxidase antibodies titer, raised TSH levels and low FT3 and FT4 level in patients with PCOS as compared to controls. Various previous researches are in agreement of current study. Similar results were also reported by $\mathrm{Yu}$ and Wang and Feldthusen et al studies who found significant high levels of TSH and thyroid peroxidase antibodies but significantly low FT3 and FT4 levels in polycystic ovary syndrome females. ${ }^{14,15}$ On contrary to current study results, previous study conducted by Saab and Haddad did not find significant hypothyroid patients among the PCOS patients. He reported no significant difference in TSH and FT4 levels in females having PCOS and controls. ${ }^{4}$

Results of current study reveal that PCOS is independent risk factor for hypothyroidism. Odd ratio of 15 obtained by logistic regression is indicating PCOS patients have 15 times more risk for getting hypothyroidism than control group.

To prevent the consequences of PCOS like infertility, the gynecologists should consider early screening of thyroid function and thyroidspecific autoantibodies at PCOS diagnosis, even in the absence of symptoms related with thyroid dysfunction.

\section{CONCLUSION}

Hypothyroidism was frequently found in PCOS patients with high TPO-Antibody titer. PCOS is an independent risk factor for hypothyroidism.

Copyright $@ 29$ Apr, 2020.

\section{REFERENCES}

1. Laganà AS, Rossetti $P$, Sapia $F$, Chiofalo $B$, Buscema M, Valenti G, Rapisarda AM, Vitale SG. Evidence-based and patient-oriented inositol treatment in polycystic ovary syndrome: Changing the perspective of the disease. International journal of endocrinology and metabolism. 2017; 15(1): e43695. doi: 10.5812/ ijem.43695.

2. Bednarska S, Siejka A. The pathogenesis and treatment of polycystic ovary syndrome: What's new. Adv Clin Exp Med. 2017; 26 (2):359-67.

3. Menon M, Ramachandran V. Antithyroid peroxidase antibodies in women with polycystic ovary syndrome. The Journal of Obstetrics and Gynecology of India. 2017; 67(1):61-5. 
4. Al-Saab R, Haddad S. Detection of thyroid autoimmunity markers in euthyroid women with polycystic ovary syndrome: A case-control study from Syria. International journal of endocrinology and metabolism. 2014; 12(3): e17954. DOI: 10.5812/ ijem.17954.

5. Delitala AP, Capobianco G, Delitala G, Cherchi PL, Dessole S. Polycystic ovary syndrome, adipose tissue and metabolic syndrome. Archives of gynecology and obstetrics. 2017; 296(3):405-19.

6. Medenica S, Nedeljkovic O, Radojevic N, Stojkovic M, Trbojevic B, Pajovic B. Thyroid dysfunction and thyroid autoimmunity in euthyroid women in achieving fertility. Eur Rev Med Pharmacol Sci. 2015; 19(6):97787.

7. Lee MW, Shin DY, Kim KJ, Hwang S, Lee EJ. The biochemical prognostic factors of subclinical hypothyroidism. Endocrinology and Metabolism. 2014; 29(2):154-62.

8. Boufas D, Vryonidou A, Mastorakos G, llias I. Thyroid function and autoimmunity versus number of pregnancies. Journal of reproduction \& infertility. 2016; 17(4):240-242.

9. de Medeiros SF, de Medeiros MA, Ormond CM, Barbosa JS, Yamamoto MM. Subclinical hypothyroidism impact on the characteristics of patients with polycystic ovary syndrome. A meta-analysis of observational studies. Gynecologic and obstetric investigation. 2018; 83(2):105-15.
10. Saran S, Gupta BS, Philip R, Singh KS, Bende SA, Agroiya $P$, Agrawal $P$. Effect of hypothyroidism on female reproductive hormones. Indian journal of endocrinology and metabolism. 2016; 20(1):108-113.

11. Singla, R., Gupta, Y., Khemani, M. and Aggarwal, S. Thyroid disorders and polycystic ovary syndrome: an emerging relationship. Indian journal of endocrinology and metabolism. 2015; 19(1), p.25-29.

12. Pushpagiri, N., Gracelyn, L.J. and Nagalingam, S. Prevalence of subclinical and overt hypothyroidism in infertile women. International Journal of Reproduction, Contraception, Obstetrics and Gynecology. 2017; 4(6), pp.1733-1738.

13. Skiba MA, Islam RM, Bell RJ, Davis SR. Understanding variation in prevalence estimates of polycystic ovary syndrome: A systematic review and meta-analysis. Human reproduction update. 2018; 24(6):694-709.

14. Yu, Q. and Wang, J.B. Subclinical hypothyroidism in PCOS: Impact on presentation, insulin resistance, and cardiovascular risk. BioMed Research International., 2016; e 2067087, doi:10.1155.2067087,

15. Feldthusen, A.D., Pedersen, P.L., Larsen, J., Toft Kristensen, T., Ellervik, C. and Kvetny, J. Impaired fertility associated with subclinical hypothyroidism and thyroid autoimmunity: The Danish General Suburban Population Study. Journal of pregnancy. 2015; e 132718, doi.10.1155/2015/132718.

\begin{tabular}{|c|l|l|}
\hline \multicolumn{3}{|c|}{ AUTHORSHIP AND CONTRIBUTION DECLARATION } \\
\hline Sr. \# & \multicolumn{1}{|c|}{ Author(s) Full Name } & \multicolumn{1}{|c|}{ Contribution to the paper } \\
\hline 1 & Anam Rehman & $\begin{array}{l}\text { Manuscript writing with its data } \\
\text { acquisition. } \\
\text { Data analysis, results } \\
\text { interpretation and drafting. } \\
\text { Drafting, Revised the } \\
\text { manuscript for intellectual } \\
\text { content. }\end{array}$ \\
\hline 3 & Shireen Jawed & Amna Rashid Tariq \\
\hline
\end{tabular}

